\title{
Aftereffects in the Perception of Emotion Following Brief, Masked Adaptor Faces
}

\author{
Nicola van Rijsbergen ${ }^{*}, 1,2$, Ali Jannati ${ }^{2}$ and Alessandro Treves ${ }^{2}$ \\ ${ }^{I}$ Department of Psychology, Glasgow University, 58 Hillhead St, G12 8QB, UK \\ ${ }^{2}$ SISSA, Cognitive Neuroscience Sector, via Beirut 2-4, Trieste, 34014, Italy
}

\begin{abstract}
Adaptation aftereffects are the tendency to perceive an ambiguous target stimulus, which follows an adaptor stimulus, as different from the adaptor. A duration dependence of face adaptation aftereffects has been demonstrated for durations of at least $500 \mathrm{~ms}$, for identity related judgments. Here we describe the duration dependence of the adaptation aftereffects of very brief $(11.7 \mathrm{~ms}-500 \mathrm{~ms})$ backwardly masked faces, on both expression and identity category judgments of ambiguous target faces. We find significant aftereffects at minimum duration $23.5 \mathrm{~ms}$ for emotional expression, and $47 \mathrm{~ms}$ for identity, but these are abolished by backward masking with an inverted face, although these same adaptors can be correctly categorized above chance.

The presence of a short duration adaptation effect in expression might be mediated by rapid transfer of low spatial frequency (LSF) information. We tested this possibility by comparing aftereffects in low pass and high pass filtered ambiguous targets, and found no evidence of independent adaptation of a LSF specific channel.
\end{abstract}

Keywords: Face aftereffects, emotion, backward masking, adaptation.

\section{INTRODUCTION}

People use the visual properties of faces to make vital social judgments about the identity, emotional state, gender, and even the trustworthiness of other people. These judgments make use of all levels of immediate visual information in the face, and also incorporate prior knowledge about both face categories, and individual faces. Recent experimental work with faces $[1,2]$ has found that category judgments can be biased by recent experience, if it includes prolonged exposure (adaptation) to an example face of a related category. This bias has been described as the 'face category adaptation aftereffect'.

How much recent visual experience is needed to produce significant bias? In the experiments we report here, we examine how the face category adaptation aftereffect depends on how much exposure to the adaptor there is in immediate perceptual history, in two different ways. First, we manipulate the exposure to the adaptor face by changing its duration [3]; previous work has shown a duration dependence only for adaptation exposures longer than 1s, while here we consider short durations, between $11.7 \mathrm{~ms}$ and $500 \mathrm{~ms}$. Second, we manipulate the amount of 'neural exposure', or the amount of processing time allowed for the adaptor by using backward masking, as described below. We also ask whether judgments about different aspects of faces depend in the same way on recent experience by comparing the effect of masking in identity and emotion judgments.

Finally, we consider the question of whether face information at low spatial frequencies (LSF; below about 8 cycles

*Address correspondence to this author at the Department of Psychology, Glasgow University, 58 Hillhead St, G12 8QB, UK;

E-mail: nicola@psy.gla.ac.uk per image [4]) is more involved in mediating the aftereffect to emotional expression, than information at higher spatial frequencies (HSF; above 23 cycles per image), as it has been hypothesized that LSF information plays an important role in rapid emotion detection.

\section{PREVIOUS STUDIES OF ADAPTATION AFTEREF- FECTS WITH FACES}

Visual adaptation aftereffects [5-7] have historically been demonstrated for simple, local visual features, such as tilt, and colour [8]. Adaptation to one stimulus (the adaptor) can change the perceived content of a following target stimulus. The induced change, i.e. the aftereffect, is consistently away from the adaptor, whereby subjects are more likely to classify an ambiguous stimulus as belonging to a different category from that of the adaptor. One explanation for these opponent aftereffects is in terms of fatigue of the neural populations encoding the adapted feature, where the exact form of the adaptation depends on the nature of the neural encoding $[9,10]$. Recently, aftereffects in the perception of natural face categories, such as identity and emotion, have been reported [1,2,11,12], as part of an emerging body of work describing aftereffects in global, high level visual features.

Aftereffects have been observed along the dimension of emotion [2], but only for relatively long exposure to the adaptor (at least one second [13]). Exposure to a fearful face (as in Fig. (1), task II) leads to a change in categorization behaviour of a following, previously ambiguous stimulus (for example, the morph target in Fig. (1)), which tends to be classified as belonging to a different category to the adaptor, i.e. neutral. Webster et al. [2] showed the adaptor before the target on every trial. Their protocol also included a phase of passive viewing of the adaptor in advance of classification 
trials. In experiment 1 we examine the contribution of a phase of pre-exposure to the magnitude of the aftereffect.

The face expression aftereffect is similar to, and may share common mechanisms with, the 'face distortion' aftereffect in the configural processing of facial identity, where altering the geometric structure of the face in the adaptor creates a misperception of the normal face structure of the target [11]. These face aftereffects have been shown to contain a component that is invariant under transformations in size [13-15] and 2D orientation [14, 16], and position [17] and therefore probably involve relatively high level, non retinopically organized cortical areas. The identity aftereffects obtained in the same fashion using 'face-antiface' pairs, defined relative to a face space norm [1], show similar size invariance and partial 3D rotation invariance $[18,19]$.

The strength of the adaptation aftereffect on the identity dimension has been shown to be duration dependent $[3,20]$ for durations longer than 1s. Almost nothing is known about the duration dependence of the expression aftereffect [20]. The experiments described in this paper show duration dependence when the durations of adaptors are short, i.e. less than $500 \mathrm{~ms}$. Aftereffects from short duration adaptors are of particular interest because it has been suggested that they are more associated with adaptation of higher level areas [2123]. In particular, a recent paper [24] found that it is the position invariant component (at least on the gender dimension) that is induced with short $(500 \mathrm{~ms})$ duration adaptors, rather than the position specific component that can be seen with longer adaptors.

Rather than focusing on which visual areas mediate the adaptation aftereffect, in our central experiment 2 we employ backward masking to try to examine the aftereffects of different temporal stages of processing of the adaptor. We refer to these short duration, masked adaptors as (negative) primes.

\section{BACKWARDLY MASKED FACES}

The perception of a visual stimulus, such as a face, can be impaired or interfered with by the presentation of a second following stimulus, such as another face. This form of interference is known as backward masking, and can result in the perceiver being unable to accurately detect, and report, the content of the first stimulus. The effectiveness of visual masking is known to depend on the Stimulus Onset Asynchrony (SOA) [25-28], i.e. the interval between the first (in this paper, the adaptor) and second stimulus (in this paper, the mask). In general, the shorter the interval between the stimulus onset, and the mask onset, the more effective is the masking. However, the mask may interfere with more than one stage of processing. At very short (less than $10 \mathrm{~ms}$ ) intervals, masking appears to block processing in the visual system completely [25]. At longer intervals (20-40ms), imaging and electrophysiological recording studies in humans suggests that early feedforward (retinotopic) stages of visual processing are largely preserved [29-31]. Comparably, in rhesus monkeys, the first few spikes $(20-30 \mathrm{~ms})$ of neural response in inferior temporal cortex are reported not to be significantly affected by the mask at an SOA of $20 \mathrm{~ms}$ [26, $27,32]$ while the later sustained response is suppressed. This may be due to the mask interrupting feedforward processing [29] or possibly disrupting a late phase of neural activity driven by re-entrant connections [30]. Face processing continues to be affected by masking up to SOAs of at least $130 \mathrm{~ms}$ [33].

The SOA is not, however, the only factor that influences masking efficacy. The content of both the mask and first stimulus is also influential. We exploit the results of [60] to adjust the content of the masking stimuli to create two masks of different strengths. Behaviourally salient stimuli, such as fearful or angry faces [34, 35] or negatively conditioned faces $[36,37]$ are still processed even when participants report no subjective awareness of the masked stimulus. We therefore chose to measure the efficacy of the two masking stimuli in reducing processing of the adaptors by our subjects' objective performance (i.e. not whether they think they saw the adaptor, but whether they can guess its content) on a forced choice task.

A number of recent studies [38-40] have reported that an early, event related potential component of face processing, between $90 \mathrm{~ms}$ post stimulus [39] to $130 \mathrm{~ms}$ post stimulus [41], is sensitive to modulation by the emotional content of face stimuli although the source of this modulation is still unknown. Face processing therefore perhaps starts relatively early, and continues to be vulnerable to interruption by a masking stimulus for a prolonged period of time, and it can be hypothesized [32] that the information build up about the stimulus is cumulative. Using the backward masking technique, we investigate in experiment 2 how quickly information about the emotional category of a face can be extracted, and how much time is needed to produce a measurable category aftereffect, on the following (third) target stimulus.

\section{SPEED OF PROCESSING OF LOW AND HIGH SPA- TIAL FREQUENCY INFORMATION}

The evidence that the emotional content of faces can be processed very rapidly $[39,42]$, and in the absence of awareness, has led to the speculation that emotional face processing is mediated by an independent fast pathway (driven by magnocellular inputs). In addition to a fast cortical route [40, $43,44]$, several authors $[35,45,46]$ have proposed the existence of a sub-cortical fast forward pathway sensitive to emotional content relayed via the colliculus and pulvinar directly to the amygdala. Evidence from evoked potentials suggests that rapid emotional processing via either route may be preferentially driven by low spatial frequency information $[40,43]$.

If a faster pathway exists for processing emotional expression information in faces, information about the emotional content of the face might be available to the subject earlier in time than information about identity, because it 'arrived faster' through a separate channel. Alternatively, making decisions about emotion might simply require less information and a shorter processing time than making decisions about identity, just because emotion discrimination is easier. These two possibilities are difficult to distinguish in behavioral experiments. In experiment 3 we examine whether the information about emotion is carried through a low spatial frequency channel.

\section{OVERALL DESIGN}

In order to measure aftereffects, we created a set of ambiguous morph stimuli (described more fully in the Appen- 
dix) from the Ekman and Matsumoto faces [47]. For the emotional expression aftereffects, we created 29 morphs from a neutral and an emotional example of the same face, whereas for the identity morphs we created 29 morphs from two neutral identities of the same gender. We took the first and last morphs as representing $100 \%$ emotion and $0 \%$ emotion, and used them as the adaptors. The 27 morphs lying between the two adaptors were used as target stimuli. For the emotion dimension, the magnitude of the aftereffect was estimated from the difference between the amount of emotion perceived in target faces after 'emotional' adaptation and the amount of emotion perceived in target faces after 'neutral' adaptation. The 'amount of emotion perceived' is measured as the proportion of 'emotional' responses at each point of a morphing continuum, spanning the 27 morph points, each point $3.44 \%$ apart, between the 'neutral' and an 'emotional' expression with the same identity. To illustrate the aftereffect we plot, for each experiment, the average proportion of emotion reported in the target against the percentage of emotion in the target along the morphing dimension.

For the identity dimension, the aftereffect was measured as the difference between the proportion of responses of identity $\mathrm{A}$ after adaptation with identity $\mathrm{B}$, and the proportion of responses of identity A after adaptation with identity A.

Our first experiment was designed to test whether adaptation aftereffects for emotional expression can be obtained with a very brief presentation of the adaptor face, subsequently masked by a blurred face of different identity (which acts as a mask of weak intensity). As we found that the aftereffect persists after a $23.5 \mathrm{~ms}$ prime, we then expanded the design to include duration as a varying parameter. We also included a stronger masking condition - when the mask is an inverted face. Experiment 2 then measures the aftereffects on the emotional continuum due to short duration adaptors, ranging from $11.7 \mathrm{~ms}$ to $300 \mathrm{~ms}$. A parallel condition measures aftereffects on identity continua in exactly the same conditions, and the same range. These parallel experiments enable us to compare the duration dependence of identity and emotional expression aftereffects.

As we find that the aftereffect to the $23.5 \mathrm{~ms}$ prime is not present on the identity dimension, one possible explanation is a faster extraction of emotional information from the masked adaptor. In experiment 3 we aimed to test the hypothesis that if there is a fast pathway for emotional processing, it is more sensitive to information carried in low spatial frequency [46]. If low spatial frequency information is critical for rapid emotion detection, the channel, or representation of LSF information may also adapt earlier than the representation of high spatial frequency (HSF) information. Our method was to use a broad spatial frequency (BSF), i.e. intact face as the adaptor stimulus, for a short duration, and then measure the aftereffects induced in the LSF and HSF 'components' of the ambiguous target, within the same subject.

We included a reference table showing all experiments and conditions (Table $\mathbf{1}$ ).

Experimental Setup. All experiments were run on a Pentium 4 computer running Windows 2000, with a CRT monitor, set to a refresh rate of $85 \mathrm{~Hz}$ using E-prime soft- ware, and data analyzed in MATLAB (7.3). Subjects were seated approximately $50 \mathrm{~cm}$ from the screen, although their head position was not constrained. All stimuli were presented $12 \times 12 \mathrm{~cm}$ wide, of which a $2 \mathrm{~cm}$ surround was a dark border superimposed over the hair contour (Fig. 1) and thus the images extended about $14^{\circ}$ of their visual fields. Of this, the actual face features occupied $7^{\circ}$.

Stimulus Duration. Exact timing of stimulus presentation was obtained with a photodiode, connected to an oscilloscope. Timing logs were also recorded during the experiment, and any trials with unusual timing errors were eliminated (about 1 or 2 per subject).

Morphed Stimuli. Gray-scale equi-luminescent 256x241-pixel stimuli were created in X-morph, from 12 face identities from the JACFEE/JACNEU Eckman series [47]. Xmorph interpolates smoothly between a grid of points, which can be fitted to the features of the face. We used a grid of approximately 100 points per face. 29 morphs were created between the neutral and emotional examples of each face. Stimuli at points 1 and 29 of the 30-point scale were used as the adaptors (the neutral adaptor and the emotional adaptor), while the remaining 27 served as target stimuli. Note that in all graphs points 1 (not 0 ) and 30 were conventionally set as containing $0 \%$ and $100 \%$ emotion, respectively, therefore the 27 target stimuli are positioned on the morphing continuum as conveying from $3.4 \%$ to $93 \%$ of the fully expressed emotion. We also performed a pilot experiment examining the emotional intensity of the morphing continua for each stimuli set (two adaptors, one neutral and one emotional, and the morphing continuum between them). This experiment is described in more detail in the supplementary material.

For experiment 2, we created a set of identity A to identity B morphs in a similar fashion, using examples of neutral expressions.

Masks. The weak mask was a quantized image made in Matlab (by setting blocks of pixels $(14 \times 14)$ to their average value) from a face that did not otherwise appear in the experiment, which had the same average luminance. The strong mask was made from the same face image as the weak mask in full detail, but inverted.

The effect of the masks at different durations was examined in a pilot experiment, which asked subjects to classify the emotion on the face of a prime of variable duration, followed either by the quantized, or the inverted mask. Our quantized mask is similar to a quantized masking condition employed by Bachmann [48], which is shown to have a strong masking effect at short durations, similar to noise masking, and a weaker masking effect at long SOAs. The inverted face mask was found in the pilot to reduce adaptor discrimination to chance at short (less than $40 \mathrm{~ms}$ ) durations, a more striking effect than previous reports have suggested [49], and to produce a stronger effect than noise at intermediate SOAS.

\section{ADAPTATION BY PRIOR EXPOSURE, AND BY SHORT AND LONG PRIMES}

\section{Experiment 1}

In this first experiment, we tested whether a reliable aftereffect could be obtained with a short duration adaptor. We 
call this adaptation by prime. Secondly, we investigated whether prior exposure to the same stimulus in another context would lead to measurably enhanced aftereffects. We call this adaptation by exposure. As an auxiliary goal, we wished to determine the best place on the emotion-neutral continuum to reliably measure aftereffects for our stimulus set.

\section{Experimental Design and Methods}

The experiment contained five different conditions. These five conditions are represented in Table 1, but in summary are (a) exposure only, (b) short prime and exposure, (c) short prime only, (d) long prime and exposure, and (e) long prime only. Our dependent measure was the magnitude of the aftereffect obtained under each condition. Statistical significance throughout the paper was considered to be $\mathrm{p}=0.05$, with non-sphericity corrections where appropriate.

Tasks. There were two tasks, a repetition detection task (inducing adaptation by exposure), and a classification task (with and without adaptation by prime), probing the aftereffect. These tasks were combined to create the five experimental conditions.

During adaptation by exposure subjects were simultaneously adapted to two randomly sequenced adaptors, one fearful (e.g. S1, "fearful Mary"), one neutral (e.g. S2, "neutral John") over 3 minutes, during task (I), represented in the top half of Fig. (1). The subject had to press the mouse every time the same identity was repeated. The stimuli were on screen for $800 \mathrm{~ms}$ each, and were separated by a mask, a fixation point, and a variable delay ( 150 or $650 \mathrm{~ms}$ ). The total viewing time for each stimulus within the exposure period was 36s. These two stimuli then served as the primes during the classification task.

In the classification task, inducing adaptation by prime, represented as task (II) in the bottom half of Fig. (1), subjects signaled (L/R mouse click) whether each target face they saw on the screen was neutral or emotional. The task was divided into blocks, one containing examples of identity S1, the second containing identity S2. For the prime conditions, an adaptor preceded each target face. This adaptor was the emotional extreme for S1 and the neutral extreme for S2. One was shown in a short prime condition, of $23.5 \mathrm{~ms}$ duration, followed by a mask of $50 \mathrm{~ms}$ duration, and one was shown in the long adaptor condition $(500 \mathrm{~ms})$, followed by the mask $(50 \mathrm{~ms})$, then after $250 \mathrm{~ms}$ the target appeared for $800 \mathrm{~ms}$, then a response screen $(1000 \mathrm{~ms})$. Subjects were instructed to ignore the first 'distracter' face stimulus (the adaptor) and the mask, and respond to the third 'target' face. For the exposure only condition the task was identical except that the adaptors were omitted, and only the mask was shown before the target.

The two tasks described above were combined to form 5 experimental conditions in the following manner. Two Exposure \& Primes conditions, (b) and (d), included the initial repetition detection task, with two adaptor stimuli, S1 and S2, followed by categorization trials with a block of long (S1) primes, and a block of short primes (S2); Exposure

Table 1. Summary of Experimental Conditions

\begin{tabular}{|c|c|c|c|c|c|c|c|c|c|}
\hline \multicolumn{2}{|c|}{ Exp. } & $\begin{array}{l}\text { Adaptor } \\
\text { (Msec) }\end{array}$ & $\begin{array}{l}\text { Mask } \\
\text { Type }\end{array}$ & $\begin{array}{l}\text { Target } \\
\text { (Filter) }\end{array}$ & $\begin{array}{c}\text { Prior } \\
\text { Exposure }\end{array}$ & Exposure Task & $\begin{array}{l}\text { Exposure } \\
\text { Measure }\end{array}$ & Aftereffect Task & Aftereffect Measure \\
\hline 1 & $\begin{array}{l}c \\
d\end{array}$ & $\begin{array}{l}\text { None } \\
23.5 \\
23.5 \\
500 \\
500\end{array}$ & $\begin{array}{l}\text { W } \\
\text { W } \\
\text { W } \\
\text { W } \\
\text { W }\end{array}$ & None & $\begin{array}{c}\text { Yes (one) } \\
\text { Yes (one) } \\
\text { No } \\
\text { Yes (one) } \\
\text { No }\end{array}$ & $\begin{array}{l}\text { Repetition detec- } \\
\text { tion }\end{array}$ & None & $\begin{array}{l}\text { Categorization of } \\
\text { morph target as emo- } \\
\text { tional face or neutral } \\
\text { face }\end{array}$ & $\begin{array}{c}\text { Difference between mean } \\
\text { emotion responses, after } \\
\text { emotion adaptor and } \\
\text { neutral adaptor }\end{array}$ \\
\hline 2 & $\mathrm{e}$ & $\begin{array}{c}11.7 \\
23.5 \\
47 \\
106 \\
306\end{array}$ & $\begin{array}{l}\text { W/S } \\
\text { W/S } \\
\text { W/S } \\
\text { W/S } \\
\text { W/S }\end{array}$ & None & $\begin{array}{l}\text { To both } \\
\text { adaptors }\end{array}$ & $\begin{array}{c}\text { Categorization of } \\
\text { masked adaptor as } \\
\text { emotional face or } \\
\text { neutral face } \\
\text { OR: } \\
\text { Categorization of } \\
\text { masked adaptor as } \\
\text { identity A face or } \\
\text { identity B face }\end{array}$ & $\%$ Correct & $\begin{array}{c}\text { Categorization of } \\
\text { morph target as emo- } \\
\text { tional face or neutral } \\
\text { face. } \\
\text { OR: } \\
\text { Categorization of } \\
\text { morph target as identity } \\
\text { A face or identity B } \\
\text { face }\end{array}$ & $\begin{array}{c}\text { Difference between mean } \\
\text { emotion responses, after } \\
\text { emotion adaptor and after } \\
\text { neutral adaptor } \\
\text { OR: } \\
\text { Difference between mean } \\
\text { identity A responses, after } \\
\text { identity A adaptor and } \\
\text { after identity B adaptor }\end{array}$ \\
\hline 3 & $\begin{array}{l}\mathrm{a} \\
\mathrm{b}\end{array}$ & $\begin{array}{l}1000 \\
\text { (BSF) }\end{array}$ & $\begin{array}{l}\mathrm{W} \\
\mathrm{W} \\
\mathrm{W}\end{array}$ & $\begin{array}{l}\text { None } \\
\text { (BSF) } \\
\text { HSF } \\
\text { LSF }\end{array}$ & Yes & $\begin{array}{c}\text { Categorization of } \\
\text { HSF and LSF } \\
\text { Morphs }\end{array}$ & & $\begin{array}{c}\text { Categorization of } \\
\text { morph target as emo- } \\
\text { tional face or neutral } \\
\text { face }\end{array}$ & $\begin{array}{c}\text { Difference between mean } \\
\text { emotion responses, after } \\
\text { emotion adaptor and } \\
\text { neutral adaptor }\end{array}$ \\
\hline 3 & $\mathrm{a}$ & $\begin{array}{l}23.5 \\
(\mathrm{BSF}) \\
11.7 \\
(\mathrm{BSF})\end{array}$ & $\begin{array}{l}\mathrm{W} \\
\mathrm{W}\end{array}$ & $\begin{array}{l}\text { HSf/ } \\
\text { LSF } \\
\text { HSF/ } \\
\text { LSF }\end{array}$ & Yes & $\begin{array}{l}\text { Categorization of } \\
\text { HSF and LSF } \\
\text { morphs and } \\
\text { masked BSF } \\
\text { adaptors } \\
\text { as neutral or emo- } \\
\text { tional }\end{array}$ & & $\begin{array}{l}\text { Categorization of } \\
\text { morph target as emo- } \\
\text { tional face or neutral } \\
\text { face }\end{array}$ & $\begin{array}{l}\text { Difference in HSF faces } \\
\text { and difference in LSF } \\
\text { faces after emotion adap- } \\
\text { tor and neutral }\end{array}$ \\
\hline
\end{tabular}

Adaptor: The duration of the adaptor is shown. Mask type: $\mathrm{W}$ denotes the weaker mask, the upright quantized face, and $\mathrm{S}$ the inverted face that acts as a stronger mask. Both masks were derived from faces that did not appear in the experiment. W/S indicates that the experiment included trials with both mask types. Target: The types of filtering of the target in each experiment. Exposure indicates whether subjects were pre-exposed to one or both adaptors prior to the trials measuring the aftereffect. Remaining columns describe the tasks and measures used. Parameters that are not included were constant for versions of the same experiment. 

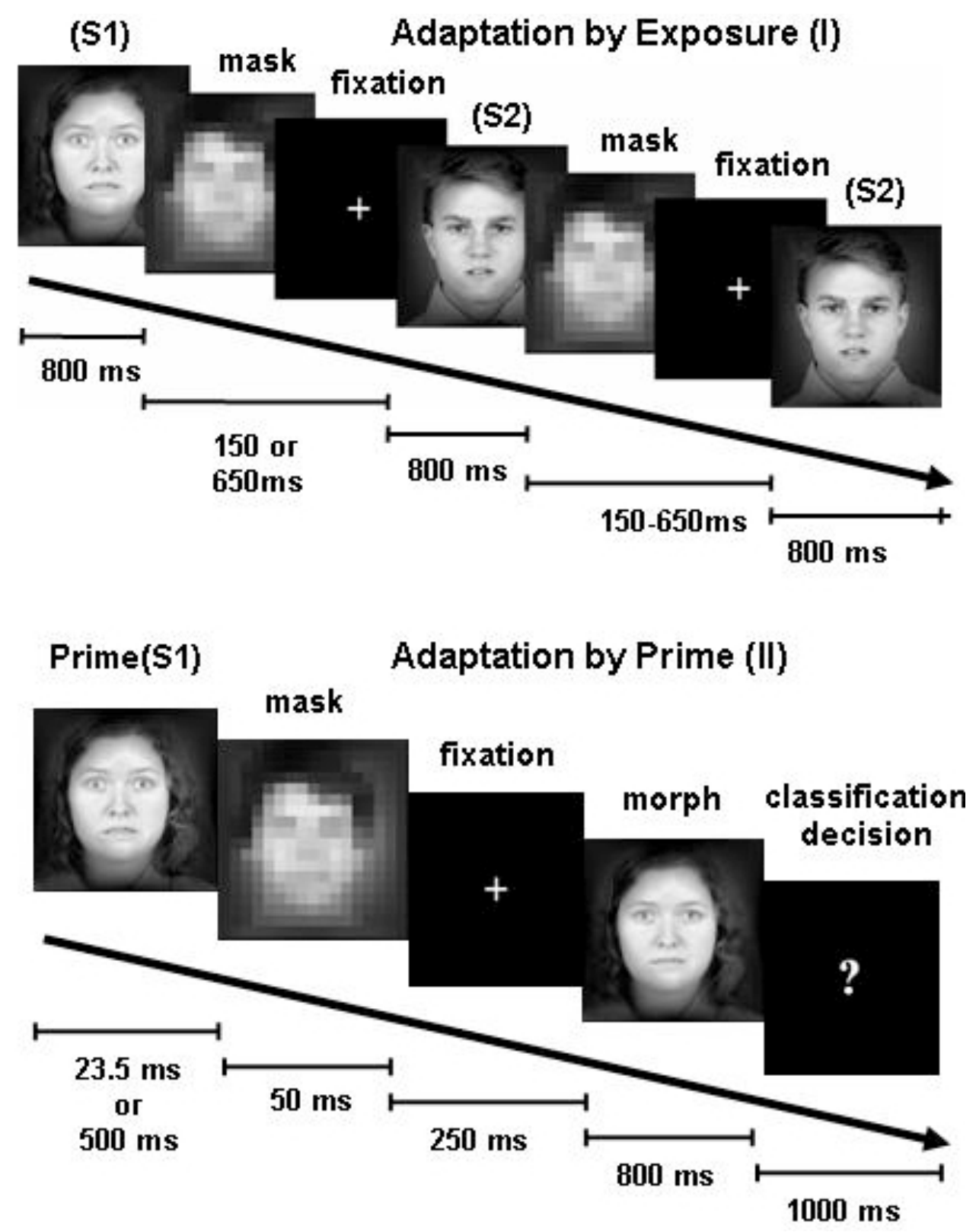

Fig. (1). Experiment 1. During the preliminary stimulus repetition task, (I), top, subjects were simultaneously adapted, by exposure, to two randomly alternating adaptors, one emotional (e.g. S1,"fearful Mary") and one neutral (e.g. S2, "neutral John"). In the classification task, (II), bottom, subjects signaled (L/R mouse click) whether each target face they saw on the screen was neutral or emotional. In the priming conditions, the target was preceded by a masked prime of duration, in conditions $1 \mathrm{~b}$ and 1d, 23.5ms (short), or 1c and 1e, 500ms (long), In the Exposure only condition (1a), the target was preceded only by the mask.

only, (a), included the repetition detection task but the categorization task contained no primes; and Prime only, (c) and (e), included separate blocks of long and of short primes, within the categorization task but omitted the repetition task. The order in which conditions were presented was counterbalanced across subjects. S1 and S2 were fully counterbalanced across subjects. Table $\mathbf{1}$ also contains a summary of the same information.

Procedure. Twenty-seven naïve subjects contributed to two experimental sessions, where stimuli (identities and emotional dimensions) were pseudo randomly assigned to the two $25 \mathrm{~min}$ sessions so that each subject contributed equally to the emotional adapted and the neutral adapted data, and categorized each identity $(\mathrm{N}=12)$ once only. Each subject performed three blocks of two categorization sets per session, and was required to rest for 2-3 minutes between blocks. Each categorization set consisted of 40 morphs from a particular image spectrum (morphs $3.4 \%-24 \%$, and $72 \%$ $93 \%$ were shown once, and the intermediate $(27-69 \%)$ ambiguous morphs twice). The valence (i.e. neutral or emotional) of the adaptor and exposure stimulus was counterbalanced across subjects, and a neutral adapted set always followed an emotional-adapted set within each block.

For each subject, we measured the magnitude of the aftereffect at the central morphs $(41 \%-62 \%)$, by subtracting the mean proportion of emotion responses after the neutral adap- 
tor, from the mean proportion of emotional responses after the emotional adaptor. We also fitted cumulative normal functions to each subject's data, and found the threshold, which is the point of inflection of the decision function, in the cumulative normal. The threshold represents the hypothetical point where a subject is equally likely to classify the target as emotional or neutral. The parametric method did not fit all subjects' data equally well, so we also employed non-parametric methods, and defined a nonparametric threshold (described in the supplementary material).
We also analyzed reaction times, as described in the supplementary material.

\section{RESULTS}

The main result of these experiments is that a reliable aftereffect could be elicited with a $23.5 \mathrm{~ms}$ adaptor, with and without prior exposure to the same stimulus.

Analysis of Aftereffects. Fig. (2) shows the change in the average of all the subjects' proportion of 'emotion' classification responses, for each possible emotion level of the
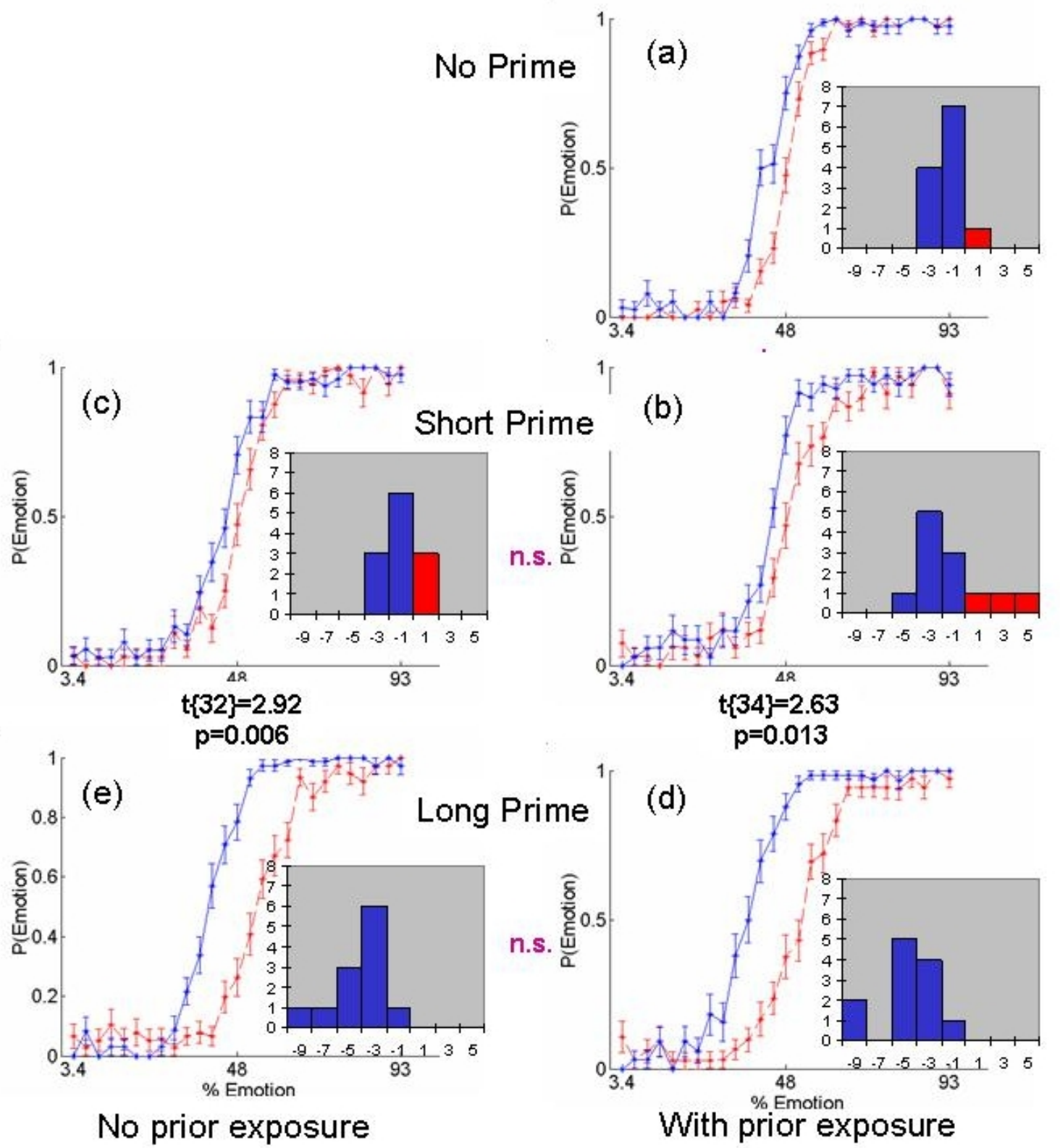

Fig. (2). Size of the adaptation aftereffects. Average proportion of 'emotion' classification responses across subjects, for each emotion level of the target (3.4\% is the most neutral target, and $93 \%$ is the most emotional), shown after emotional adaptation (red), and neutral adaptation (blue). Each condition is shown separately, with the exposure conditions on the right, and the prime only conditions on the left. Individual Conditions 1a-1e: The 5 conditions tested differ by the absence (left column) or presence (right column) of a prior exposure adaptation phase; the absence of a priming face (top row); the presence of a $23.5 \mathrm{~ms}$ prime (middle row) or of a $500 \mathrm{~ms}$ prime (bottom row). Error bars +/- are S.E.M. Inset in each graph is the histogram of average threshold changes for the 12 stimuli sets, estimated with the parametric method. 
target, after emotional adaptation (red) and neutral adaptation (blue). Each condition is shown separately, with the exposure conditions on the right, and the prime only conditions on the left.

A 2-way ANOVA (5 conditions, and adapting end-point) of the frequency of responding emotional to the seven most ambiguous morphs (the central morph $41 \%-62 \%$ ) found an effect of adaptation direction, $F\{1,200\}=123, p<0.0001$, $M S E=4.60$, and a significant interaction between adaptation direction and condition, $F\{3,200\}=7.41, \quad p=0.0001$, $M S E=0.28$. Significant aftereffects appeared in all five conditions. Neutral adapted - Emotional adapted contrasts were significant for Long Prime, $F\{1,34\}=34.1, \quad p<0.01$, $M S E=1.52$; Short Prime, $F\{1,34\}=7.12, p<0.05, M S E=0.26$; Exposure \& Long Prime, $F\{1,30\}=44, p<0.0001, M S E=1.71$; Exposure \& Short Prime, $F\{1,34\}=11.15, \quad p=0.002$, $M S E=0.39$; Exposure only, $F\{1,37\}=4.7, p<0.04, M S E=0.12$.

The effect of condition was essentially a difference between the two long adaptor conditions (d, e), and the other three. There were no statistical differences between the two $500 \mathrm{~ms}$ prime conditions, nor between the $23.5 \mathrm{~ms}$ prime conditions, or between the latter and exposure only. This suggests that the exposure period had no additional effect above that of the prime. Long and short primes produced different magnitudes of aftereffect, determined by comparison of the difference between emotional adapted and neutral adapted in each condition. We used the $t$-test to examine the difference in the magnitude of the adaptation aftereffects between pairs of conditions. The differences between long and short primes were significant: Exposure \& Long Prime versus Exposure \& Short Prime: $t\{32\}=2.92, p<0.03$; Long Prime versus Short Prime: $t\{34\}=2.63, p=0.05$, Bonferroni corrected for 5 tests.

Distributions of parametric threshold values are shown in the insets of Fig. (2). Each inset shows a histogram of the average (across subjects) of the threshold shifts for the 12 stimuli. The threshold shifts of individual stimuli were not significantly correlated across conditions.

\section{DISCUSSION}

We established that a measurable aftereffect can be obtained with the presentation of a $23.5 \mathrm{~ms}$ prime, masked with a weak mask, and that the magnitude of this aftereffect appears to depend on the duration of the preceding prime, and not on the presence or absence of pre-exposure. This is different from results reported by Hsu and Young [13] who did not obtain a reliable aftereffect in emotional expression with $500 \mathrm{~ms}$ adaptors, but who used a very long (1 second) interval between adaptor and target.

The large aftereffects obtained with long primes presumably reflect a variety of conscious processes, involving the careful examination of the adaptor and the retention of relevant information in short term memory. Our data also suggest that in the absence of an immediately preceding adaptor of sufficient duration to allow conscious comparison with the target face, the aftereffects induced by prior exposure and by short adaptors are all of similar magnitude. In particular, adaptation by prior exposure does induce a measurable aftereffect, which appears to be stimulus-specific and to survive the time interval (approximately $2 \mathrm{~min}$ ) between the exposure session and the main task (including all the intervening stimuli). However, it does not add to the short term effect of the prime, even if the effect of the prime is small.

Subject debriefing afterwards suggested that subjects paid little attention to the masked stimuli, and were unaware of the content of the short prime. However, the subjects in an independent pilot experiment performed significantly above chance across trials (mean 69\% + STD +/-11\%), when single stimuli were presented for $23.5 \mathrm{~ms}$ and masked by the quantized (weak) mask (see supplementary material).

\section{STRONGER MASKING, AND IDENTITY VS EMO- TION}

\section{Experiment 2}

A pilot experiment had shown that masking with an inverted face, i.e. a stronger mask, at the same adaptor duration $(23.5 \mathrm{~ms})$, reduced discrimination of the adaptor content to chance level. With this result in mind, we designed the next experiment to examine the effects of stronger backward masking' on short duration stimuli.

The motivation for experiment 2 was two-fold. Firstly, we wanted to describe the dependence of the aftereffect on the duration of the adaptor on both identity and emotion adaptation. Secondly, we wanted to examine the effect of masking of the adaptor on the aftereffect, by using a mask that reduced subject's ability to explicitly categorize the adaptor content.

We hypothesized that masking may disrupt identity adaptation at durations where emotional adaptation is still preserved, as information about the emotional content of the adaptor may be processed more rapidly than identity information. Thus, if the mask stops processing at the right point, we should be able to observe adaptation to the emotional category but not to identity. To test the latter hypothesis, we chose to compare adaptation obtained with the mask used in experiment 1 (our weak masking condition) with a mask consisting of an inverted face of the same average luminance (a strong masking condition). We also measured objective categorization of the masked adaptor at each duration in a separate block.

In order to describe the duration dependence of identity adaptation and emotional adaptation, we conducted two parallel experiments with the same procedure, an emotion condition inducing emotional adaptation, and an identity condition, inducing identity adaptation. The experimental procedure used the same set of durations, and two different masks for both conditions. To do this we created a second set of morph stimuli spanning four continua between four pairs of adaptor identities ( $\mathrm{A}$ and $\mathrm{B}$ ), where one of the identities was the neutral adaptor from the emotion experiment. Obviously identity A / identity B morphing continua do not form a 'natural' spectrum, and subjects require some prior experience or training to be able to successfully identify unknown faces. We therefore introduced a training phase into the experimental design, for both the emotion/neutral and the identity/identity experiments. During this phase, we measured the subjects' accuracy at classifying examples of the adaptors at different durations, and under different masking conditions. This enabled us to examine the relationship between 
each individual subject's ability to objectively classify adaptors, and their bias during the aftereffect trials.

\section{Experimental Design and Methods}

The two experimental factors, adaptor duration, and mask - strong (inverted) versus weak (quantized) - were varied in exactly the same manner in the two experiments. The two shortest presentation times were $11.7 \mathrm{~ms}$ (one screen refresh) and $23.5 \mathrm{~ms}$ ( 2 screen refreshes). Additional durations of $47 \mathrm{~ms}, 106 \mathrm{~ms}$, and $305 \mathrm{~ms}(4,9$ and 26 refreshes) were also used.

Subjects. 19 students from the University of Trieste participated in the emotion/neutral adaptation condition, and 20 students participated in the identity/identity condition.

Procedure. Each subject performed 4 blocks of trials, order counterbalanced across subjects, where each block included a different pair of adaptors, and a set of morphs taken from the morphing continuum. In the identity A to identity B continuum, the two adaptor faces were of the same gender and neutral expression. In the emotional to neutral continua, the two faces were of the same identity, one was always neutral, and one was fearful or angry.

Each block comprised a training phase and an adaptation phase. In the identity experiment the training phase consisted of 20 trials as follows: the subject was presented with a training screen for $3 \mathrm{~s}$ containing a pair of identities, with associated names e.g., 'Anna' on the left of the screen, and 'Lucy' on the right side of the screen. The screen also assigned Lucy or Anna to two letters on the keyboard, a right (n) or left key press (x), which corresponded to their screen position, counterbalanced across subjects. Two seconds afterwards they were presented with a masked stimulus at one of the five durations, and asked to guess the identity of the stimulus before the mask, and to respond with the corresponding $\mathrm{x} / \mathrm{n}$ key choice. The training screen with the key assignments preceded every trial until they had seen two examples of each mask/duration combination for that identity pair.

The subject then performed 80 adaptation trials in random order, 2 adaptors (e.g. Lucy or Anna) at five durations, and with 2 masks. Here they were asked to classify the target following the masked stimulus. Targets were shown for $300 \mathrm{~ms}$, and were morphs from the central section of the continuum, levels $(41 \%, 48 \%, 54 \%$ or $62 \%)$.

The emotion condition was designed and run in exactly the same manner, except that the training screen in each block contained examples of a neutral expression, and a fearful or angry expression of the same identity, presented side by side, and labeled 'fear' and 'neutral', or 'angry' and 'neutral' with associated key responses.

For the training data, we calculated the percent correct at each mask/duration combination. We also measured each subject's average reaction time for each mask and duration combination. For the aftereffects, each subject's average proportion of response 'emotion' and average response 'identity A' was computed for each adaptor, mask and duration combination. We took the magnitude of the difference between the two adaptation directions, at each mask and duration, for each subject, and then averaged across subjects. We also computed correlations between percent correct scores and adaptation magnitudes for each duration/mask combination, both on average, and across the population.

\section{RESULTS}

The left panels of Fig. (3) show how accuracy of the objective classification of the adaptor increases with duration in both identity and emotion experiments. The right hand panels show how the mean magnitude of aftereffects in the two conditions also increases. There is a clear difference between the weakly masked (dashed lines) and the strongly masked conditions in both tasks of both conditions. The main differences between the two experiments are summarized in Fig. (4), where there is a clear aftereffect at $23.5 \mathrm{~ms}$ in the emotion experiment for weak masking that is not present in the identity experiment and an absence of an aftereffect in the strong masking condition for identity, at $305 \mathrm{~ms}$.

Identity versus Emotion. Subjects were significantly more accurate on the emotion classification task, overall, than on the identity task. Separate ANOVAs, for each mask type, with two factors, experiment (identity or emotion) and duration, showed an effect of experiment on the classification task, in both the quantized mask $F\{1,37\}=17.38$, $p<0.001, M S E=0.48$ and in the inverted mask $F\{1,37\}=5.02$, $p<0.03, M S E=0.18$, as well as strong effects of duration, $F\{4,34\}=19.44, \quad p<0.005$ and $F\{4,34\}=24.12, \quad p<0.005$. However, comparison of the magnitudes of aftereffects between experiments showed no overall difference between identity and emotion, although there was still a strong effect of duration, $F\{4,34\}=11.8, \quad p<0.01$ and $F\{4,34\}=4.13$, $p<0.05$. Comparison of aftereffect magnitudes in the two experiments is hard to interpret, due to potential scaling issues. To capture the effect of duration, therefore, we divided the data into the two experimental conditions and analyzed them separately with within-subject ANOVAs.

Emotion. Within subjects, however, there was a striking difference between the two mask types in both tasks. In the emotion experiment, a within subjects ANOVA, on the classification task, found effects of mask type, $F\{1,18\}=23.02$, $p<0.0005, M S E=0.76$ and duration $F\{4,72\}=17.17, p<0.005$, $M S E=0.57$. The same analysis on the adaptation task found an effect of duration, $F\{4,72\}=12.75, p<0.005$, MSE $=0.34$, and mask type $F\{1,18\}=10.81, p<0.005, M S E=0.44$.

In the classification task (Fig. 3, bottom left) subjects were on average more accurate for weak than strong masking. Post hoc paired t-tests found a reliable difference between the two masking conditions at $23.5 \mathrm{~ms}, p<0.01$, and otherwise the difference was approximately constant, but not significant at individual durations.

The aftereffect (Fig. 3, bottom right), was also greater for the weak masking condition than the strong masking condition, but the effect of duration much less on the strong masking condition. In order to understand the effect of durations on the aftereffect in each masking condition, we conducted four paired $t$-tests comparing successive points for each masking condition, looking for the largest transition between successive durations. We also compared the largest increases with chance (zero bias). Adaptation effects for the weak mask show the largest increase when we compare $11.7 \mathrm{~ms}$ with $23.5 \mathrm{~ms}$ ( $p<0.05$, Bonferroni-corrected for 5 tests), and the aftereffect at $23.5 \mathrm{~ms}$ is also different from chance (one 

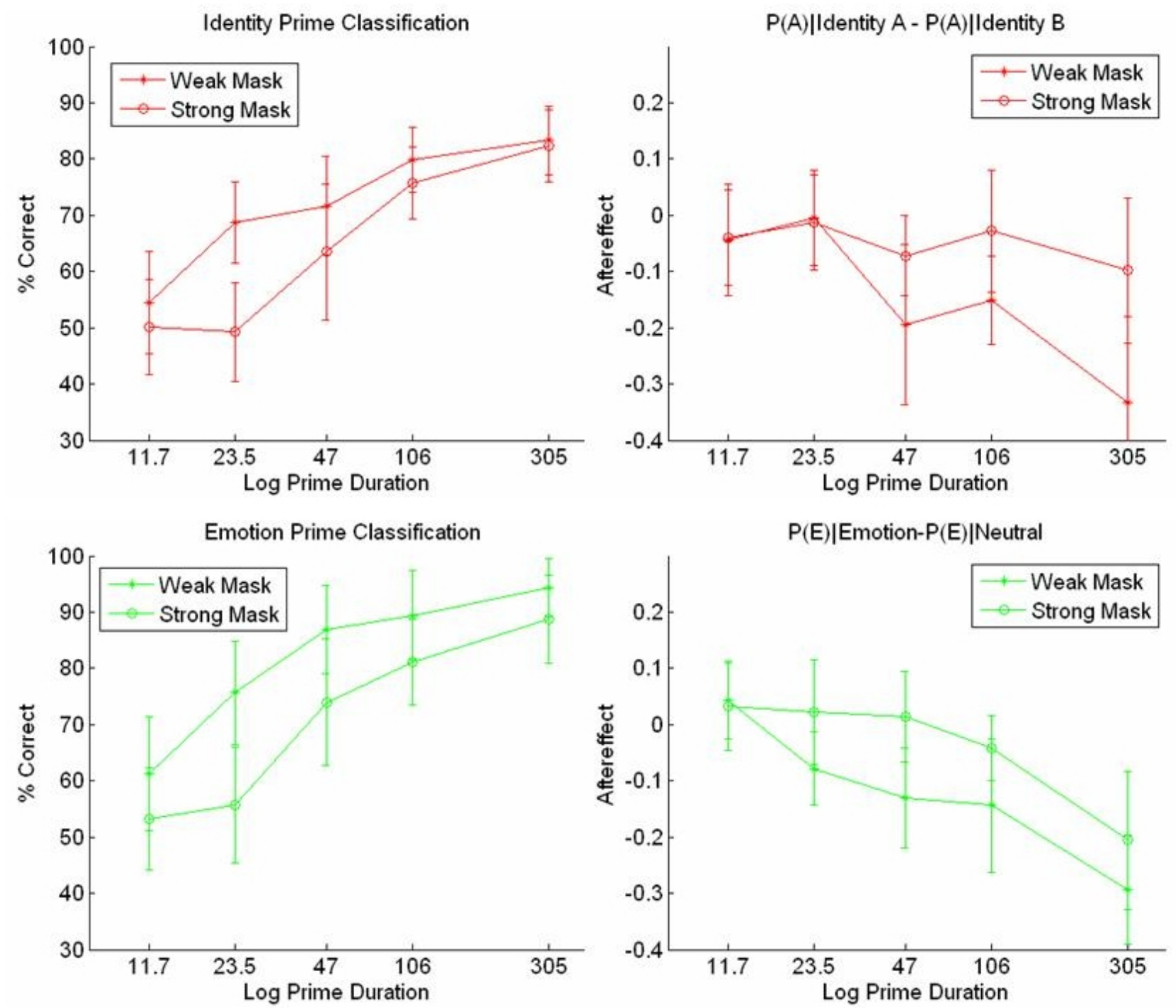

Fig. (3). Duration dependence of prime identification, and of the resulting aftereffect. The results of the emotion condition are shown in the lower graphs, and the results of the identity condition in the upper graphs. Average percent correct adaptor classification (left) and average aftereffect magnitude (right) plotted against adaptor duration on a log scale. In the identity condition (top) the aftereffect magnitude is the difference between (the fraction of) identity A responses, after the A adaptor, and identity A responses after the B adaptor; while for the emotion condition (bottom) it is the average of the difference, for each subject, between emotion responses after the emotional adaptor, and after the neutral adaptor. In each experiment, the weak masking condition is shown as crosses and the strong masking condition as circles. Error bars are confidence intervals.

tailed $t$-test, $\mathrm{p}=0.05$, Bonferroni-corrected) and there is a trend towards a change between $106 \mathrm{~ms}$ and $305 \mathrm{~ms}$, that does not meet the correction level ( $p=0.02$, uncorrected). For the strong face mask, the adaptation aftereffect becomes significantly different from chance at $305 \mathrm{~ms} \quad(p<0.002$, Bonferronicorrected for 5 tests), but none of the transitions are significant. In particular, the largest transition from 106ms to $305 \mathrm{~ms}$ is not significant ( $p=0.026$, uncorrected), suggesting that the aftereffect is already there at $100 \mathrm{~ms}$ in some subjects.

Identity. In the identity condition, the effect of duration on accuracy in the classification task was larger than for the same task in the emotion experiment, $F\{4,76\}=21, p<0.001$, $M S E=0.64$ and the effect of mask type smaller $F\{1,19\}=9$, $p<0.003, M S E=0.27$, with a trend towards an interaction,
$F\{4,76\}=1.91, p=0.1$. In the adaptation task, the effects were similar to emotion for mask type $F\{1,19\}=8.6, p<0.001$, $M S E=0.45$, and smaller for duration $F\{4,76\}=5, p<0.004$, $M S E=0.26$.

The classification data (Fig. 3, top left), shows that subjects are more accurate in the weak masking condition than the strong masking condition, but at $305 \mathrm{~ms}$ the difference disappears. Pair-wise comparisons between the two masking conditions at matched durations, established that, on the classification task, subject accuracy in the two mask conditions differs reliably only at $23.5 \mathrm{~ms}$, where it is above chance with the weak mask, $t=2.81, p=0.01$ and at chance with the strong mask. Percent correct data in the strong mask condition is above chance, $t=2.17, p=0.03$ at $47 \mathrm{~ms}$. 

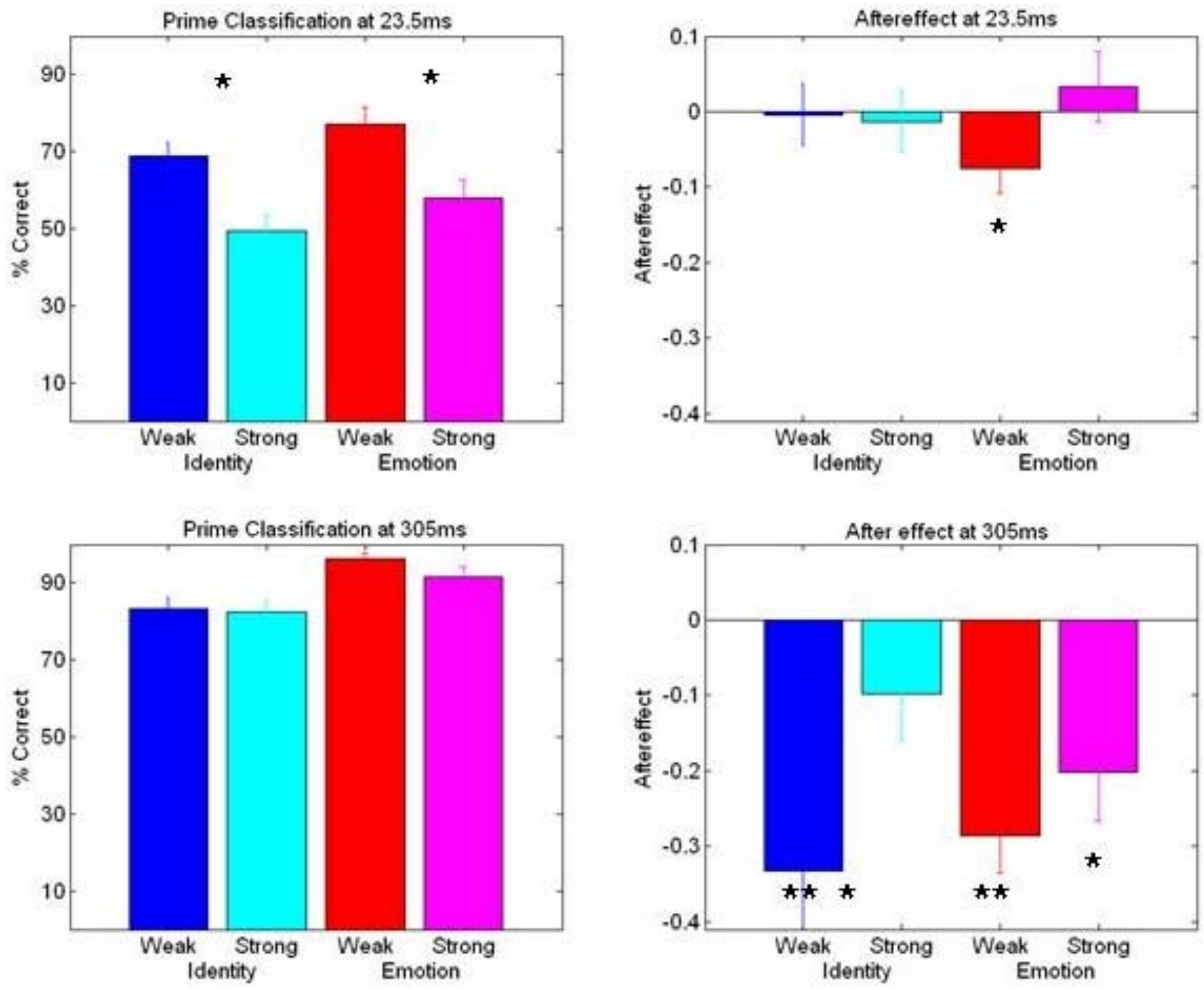

Fig. (4). Summary of experiment 2. The average percent correct on the classification task (left) is shown in both masking conditions at $23.5 \mathrm{~ms}$, and at $305 \mathrm{~ms}$, for both experiments. The strong and weak masking conditions are significantly different from each other in both the identity and emotion experiments at $23.5 \mathrm{~ms}$ (top left) but not at $305 \mathrm{~ms}$ (bottom left). On the top right, the average aftereffect is significantly different from chance at the bonferroni corrected level 0.05 , at $23.5 \mathrm{~ms}$ only in the weak masking condition in the emotion experiment. On the bottom right, at $305 \mathrm{~ms}$, in the emotion experiment, both masking conditions show aftereffects different from chance, and are not different from each other. In the identity experiment, only the aftereffect in the weak masking condition is significantly different from chance. It is also different from the strong masking condition. * indicates $\mathrm{p}<0.05, * *$ indicates $\mathrm{p}<0.005$, corrected for five tests.

In the adaptation task (Fig. 3, top right), we found no significant pairwise transitions. A reliable aftereffect appears at $47 \mathrm{~ms}$, for the weak mask, and reaches the Bonferronicorrected level of significance, $p=0.05$ (compared to chance, one-tailed); whereas the aftereffect, in the strong masking condition at $305 \mathrm{~ms}$, only reaches a significance level of $p=0.05$, uncorrected (also compared to chance, one-tailed). This pattern of results again suggested that it was a subgroup of subjects who were driving the aftereffect in the strong masking condition, and that this group might already show aftereffects at $106 \mathrm{~ms}$ (even though the group is still at chance, as can be seen in Fig. (3)). This proved to be the case, as aftereffects were strongly correlated within subjects, correlation coefficient $r=0.48, p<0.03$ at these durations.

Reaction Times. We analyzed the reaction times of subjects' responses on both tasks. Only the classification task showed any effects of duration, mask, or experiment. Subjects were faster on the emotion classification task, than identity: rank sum test, $p=0.03$. This was due to a difference between the two full masking conditions $(p=0.007)$, as the median RT's for the weak masking conditions were not significantly different, $p=0.43$. Again, we also analyzed the two experiments separately. Overall, subjects were slower to respond to the strongly masked adaptors, in both experiments.

Further analysis, i.e. an ANOVA on reaction times for the emotion experiment for random effects, with duration and mask as factors (thus neglecting the non-normality), showed that there was an effect of duration $F\{1,18\}=11.5$, $p<0.03$, and mask $F\{4,72\}=12.65, p<<0.005$. Identical analysis of the Identity experiment found larger effects of masking $F\{1,19\}=68.7, p<<0.0005$, and a smaller effect of 
duration $F\{4,76\}=9.58, p<0.005$. In both experiments, subjects took longer to respond to the shortest stimuli, 11.7 and $23.5 \mathrm{~ms}$, in both conditions. Two Friedman's ANOVAs confirmed these effects. Overall the slowest reaction times were for strongly masked identity trials.

Comparison of the Two Tasks. Finally, we were interested in whether performance on the two tasks was closely related, both at an individual, and at a group level.

Our hypothesis was that aftereffect would depend on duration. Using ANCOVA, we found that the aftereffect magnitude depended on duration $F\{1,12\}=53, \mathrm{p}<.001$ once group means $F\{3,12\}=6.17$ were taken into account, with a borderline interaction term $F\{3,12\}=3.01, \mathrm{p}=0.07$. The accuracy results also depended on duration, $F\{1,17\}=25$, $\mathrm{p}=0.002$, best fit by the same relationship for all the groups $F\{3,13\}=3.35, \mathrm{p}<0.05$. Performance on both tasks clearly depends on a common variable, duration. We were also interested in the relationship between performance on the classification task and the aftereffect. An ANCOVA using accuracy as the covariate, found that accuracy was a predictor of aftereffect magnitude $F\{1,18\}=17, p=0.006$, and group differences were no longer significant.

The relationship between accuracy on the classification task and aftereffect magnitude only held at the level of the group means. Analysis of individual subject data found significant correlations in individual performance between these two tasks only in one of the four groups, the identity experiment, with strong masking condition, at the duration of 106 $\mathrm{ms}, r=-0.45, \mathrm{p}=0.06$, and the duration of $305 \mathrm{~ms}, r=-0.42$, $p=0.04$. The same sub group of subjects that showed high accuracy on the classification task, also showed evidence of aftereffects (i.e., negative bias) at both durations, even though the group on average showed no aftereffects, as shown in the bottom right of Fig. (4).

\section{DISCUSSION}

In summary, the data suggest that the magnitude of the adaptation effect for both identity and emotion depends on duration in much the same manner. However, information about emotion category is available at shorter durations. Emotional category decisions might not, however, be made faster, as reaction times are not significantly different across the identity and emotion experiments for the partial masking condition. In the strong masking condition of the identity experiment, decisions are made slower, and are less accurate than in the strongly masked condition of the emotion task. Subjects also took longer to respond to the short duration stimuli, and longer to process the fully masked stimuli. This is consistent with the idea that subjects are using longer processing times to attempt to compensate for reduced visibility of stimuli, whether reduced by short duration times, or severe masking [25].

In addition, strong masking interferes with the aftereffect in both identity and emotion, almost completely abolishing the aftereffect in identity. The aftereffect in the emotion task appears at a shorter duration $(23.5 \mathrm{~ms})$, in the partially masked condition. In the identity condition, at that duration, there is not even a trend. The observed relationship between accuracy and aftereffect suggests that 'decoding' the neural response plays a substantial role in determining aftereffect magnitude. It might have been the case that aftereffect strength was most closely related to the duration of the adaptor, indicating that the aftereffect was driven by adaptation to an early, arguably feedforward phase, of stimulus processing. Instead, we find that full masking interferes with the aftereffect, abolishing it completely in the identity experiment, even up to a duration time of $300 \mathrm{~ms}$, as illustrated by Fig. (4, bottom right) even though the subjects' ability to categorize the adaptor is equivalent to the partial masking case, (shown in Fig. (4), bottom left).

Overall, our results on the classification support the idea that information about emotion is in part extracted prior to information about individual identity. We also find that strong masking disrupts the emotional expression aftereffect. We therefore suggest that emotional classification is perhaps possible on the basis of a stage of stimulus processing that is preserved in backward masking (perhaps the stages $<110 \mathrm{~ms}$ after stimulus onset $[30,31])$. In contrast, the aftereffect may result from adaptation of a later neural process, which is thus vulnerable to interference from a masking stimulus. The content of the mask may also have played an important role; the choice of another face, albeit inverted, may have promoted competition for neural representation in the same region [50].

Our results establish that there is rapid adaptation to emotional content in faces, but there is still an open question about the nature of the channel through which emotional information is extracted. It has been argued elsewhere that there is a fast processing pathway for emotions, via the pulvinar to the amygdala. Information derived from this pathway could be sufficient to give category information about emotional expression, and could be driving aftereffect induction at short durations. One putative characteristic of this pathway is that it is more sensitive to information in the low spatial frequency range. This motivated us to investigate whether the aftereffects were expressed independently in High Spatial Frequency (HSF) and Low Spatial Frequency (LSF) representations of the same face.

\section{ADAPTATION OF LOW AND HIGH SPATIAL FRE- QUENCY CHANNELS}

\section{Experiment 3}

Experiment 3 was designed to test the possibility that a fast emotional pathway preferring LSF information was driving the aftereffect at the shortest duration $(23.5 \mathrm{~ms})$ at which we reported significant effects for emotional expression. We also included a shorter duration $(11.7 \mathrm{~ms})$, in case $23.5 \mathrm{~ms}$ was insufficiently brief for channel specific effects. We chose to use a Broad Spatial Frequency (BSF, i.e. unfiltered) adaptor, and to look for effects in HSF and LSF faces derived from that adaptor. If LSF information was being used to drive the category decision, we expected to find more adaptation in the LSF face target, and for the magnitude of the aftereffect to be uncorrelated with the magnitude of the aftereffect expressed in HSF targets. Previous work, for example [12], has concentrated on whether adaptation can be induced independently by HSF and LSF adaptors. They found that configural face adaptation in HSF and LSF channels is highly specific, and that aftereffects do not survive changes in spatial frequency. 
HSF and LSF information is thought to be processed through largely independent channels, at early stages in the visual system, and presumably integrated into a single percept before reaching higher cortical areas. It is hypothesized that the amygdala and connected sub-cortical systems preferentially process low frequency information [51-53]. In Vuilleumier, Armony et al. (2003) [53], the responses of sub cortical and cortical regions to a HSF or LSF face image were measured after 'priming' with HSF and LSF versions of the same image. Repetition suppression in the BOLD signal in the fusiform gyrus was reported for LSF faces with HSF primes, but not vice versa. This asymmetrical transfer may suggest that cortical processes establish repetition suppression (a form of adaptation), subsequently affecting also sub-cortical processes. It thus remains unclear how independent the processing of HSF and LSF information about high-level properties might be. Among face selective neuronal populations tuned to different spatial frequency components, some may be distinct cortical populations [54], while others may reflect the cortical / sub-cortical divide [52, 54]. Notably [40, 43] found LSF fearful faces modulated the P100, an early component of visual processing, while HSF faces did not.

Our approach differs from both [12] and [51] as we examine the results of adaptation by an intact (BSF stimulus), on its HSF and LSF components. We thus consider the question of whether, in the perception of natural face images, LSF information and HSF information adapt independently and whether more rapid extraction of LSF information, means that at short adaptor durations, adaptation is expressed more strongly in LSF components.

If our weakly masked short duration adaptors only permitted LSF information to be extracted, we might expect uncorrelated adaptation to expression in the HSF and LSF representations, and significantly less adaptation of HSF face representations. An alternative possibility is that the aftereffect is induced by adaptation that occurs at a stage after integration of HSF and LSF information. In this case, adaptation should either be seen in both, or neither.

\section{Experimental Design and Methods}

In order to first examine whether BSF images adapted their HSF and LSF counterparts, we conducted a preliminary experiment, using a long duration adaptor (1s), and examined the sizes of the aftereffects induced, and the shifts in the decision function for each morph set. Then we conducted experiments with short duration adaptors, one $23.5 \mathrm{~ms}$, and one $11.7 \mathrm{~ms}$, and measured aftereffect sizes. Both experiments followed the same procedures, except for a change in baseline measurement, and used the same stimuli set. For the $23.5 \mathrm{~ms}$ and $11.7 \mathrm{~ms}$ adaptation conditions, we also included 20 trials asking subjects to classify the content of the masked adaptors, as part of a pre-training block (similar to the classification tasks in experiment 2).

Stimuli. Spatially filtered stimuli were derived from four morph sets. The images were filtered in Matlab with a 2dimensional Butterworth filter, at a range of cutoff frequencies, and the HSF images at the endpoints of the continua (not the morphs) were also weakly smoothed with a gaussian filter to simulate the loss of high frequency components observed to result from the morphing process. The lumines- cence of the images was then renormalized. Psychophysical testing, aimed at balancing the difficulty of discrimination within the high and low pass filtered morphs, led us to select cut off frequencies of 12 cycles per image (LSF), and 40 cycles per image (HSF; see Fig. (6)) consistent with the ranges of cutoff frequencies that support identity judgments in other experiments [55]. Subjects were $50 \mathrm{~cm}$ from the screen and the stimuli were $12 \times 12 \mathrm{~cm}$ wide $(256 \times 241$ pixels), with the face image occupying the central $7 \mathrm{~cm}$, resulting in a LSF cut off of approximately 0.8 cycles per visual degree, about 6-7 cycles per face. HSF stimuli had a cutoff about 23 cycles per face. Pilot study subjects $(\mathrm{N}=20)$ asked to categorize the end point targets achieved accuracy levels of $73 \%$ (LSF) and at $82 \%$ (HSF), which were not significantly different.

Procedure (1s Adaptor). Subjects first classified a baseline block as containing all of the target stimuli, both HSF and LSF, as either emotional or neutral expressions, with no adaptation.

Subjects then classified targets in four separate blocks (order counterbalanced between subjects). Each block contained both high frequency and low frequency versions of faces sampled from a particular continuum, preceded by the BSF version of the face (the adaptor). Blocks were constructed as follows. The adaptor was shown for ten presentations; then four target trials in random order, as Adaptor, followed by HSF, Adaptor followed by LSF, Adaptor followed by LSF and then HSF, or Adaptor followed by HSF, then LSF. The sequence of trials then repeated. There were 60 trials per stimulus block comprising HSF and LSF morphs from positions 10-20 (34\%-68\%) inclusive, and 16 HSF \& LSF versions of the endpoints (3.4\% and 93\%). Adaptors and targets were shown for one second. Target morphs and endpoints were randomly sorted, and each subject saw each morph at least twice, once directly after the adaptor, and once following one intervening target of the other spatial frequency.

Procedure (23.5ms, and $11.7 \mathrm{~ms})$. The procedure for the conditions with brief adaptors $(23.5 \mathrm{~ms}$, and $11.7 \mathrm{~ms})$, was essentially the same, except that the stimulus blocks were lengthened to 80 adaptor trials, with each target preceded by an adaptor, and the trial order fully randomized. 20 baseline (no adaptor) trials were included within the blocks. Subjects still classified a pre-training block containing all the stimuli, but these responses were discarded.

Subjects. Fourteen subjects saw 1s adaptors, Fourteen subjects saw $23.5 \mathrm{~ms}$ adaptors, and seventeen subjects saw $11.7 \mathrm{~ms}$ adaptors. Six subjects also performed the same procedure with the same proportion of prime to test stimuli, on full-spectrum faces, thus enabling us to compare HSF and LSF adaptation expression to the BSF adaptation expression between subjects.

Analysis. Aftereffects were measured by the average difference in proportion of the response 'emotional' following the emotional adaptor, minus the proportion of response 'emotional', following the neutral adaptor. In the 1s adaptor condition we initially analyzed the responses preceded by the adaptor separately from responses where there was an intervening stimulus, but found that there was no difference between them. For further analyses we collapsed across these two sorts of trials. We also fitted nonparametric thresholds to 
the responses to spatially filtered, HSF and LSF targets, and unfiltered BSF targets. The final part of the analysis examined the correlation between the expression of the aftereffect in HSF and LSF targets, within individual subjects.

\section{Results: 1s Adaptor}

The graphs of Fig. (5) show both the baseline (a) responses to HSF and LSF targets, and then the decision functions to HSF (a), LSF (b), and BSF (c), after emotional and neutral adaptation. In each case, there is a clear difference between the two decision functions. We found significant aftereffects in three-way ANOVAs (morph level, and adaptor type, subject) for each morph set: $\operatorname{HSF}(F\{1,13\}=79$, $p<0.0001, M S E=10.8)$ and $\operatorname{LSF}(F\{1,13\}=18.26, p=0.02$, $M S E=2.5$ ). Average aftereffect (Neutral adaptation - Emotional adaptation), within subjects, was greater than zero for HSF (mean difference $+/$-standard deviation, $0.27+/-0.21$ ), $t\{13\}=4.9, \quad p<0.02)$; and LSF $(0.12+/-0.17, \quad t\{13\}=2.4$, $p<0.05)$. The average difference between the aftereffects in HSF and LSF sets was also significant $(0.15+/-0.19$, $t\{26\}=2.11, p<0.05)$. We also analyzed the data from the six subjects who saw the BSF targets, and found significant aftereffects, BSF $(F\{1,5\}=30, p<0.05, M S E=3.9)$ which were of intermediate size and not significantly different from the effects in the LSF targets.

The non parametric threshold difference was largest for HSF morphs (21\% units), smaller for BSF (14\% units) and much smaller for LSF stimuli (3.4\%). It is noticeable that the thresholds for both emotional and neutral-adapted LSF stimuli slightly shifted towards the emotional, relative to baseline. The accuracy of classification of the endpoints also increased relative to baseline in both emotional and neutral adaptor conditions. Both changes probably reflect increasing subject familiarity with the face set.

We also examined the extent to which adaptation of low and high pass filtered stimuli was correlated within the responses to each stimulus for each of the fourteen subjects. We compared the difference between an individual subject's mean proportion of classification as 'emotional' before and after adaptation, for each morph set. Neutral adaptation expressed in the HSF morph set was weakly but significantly
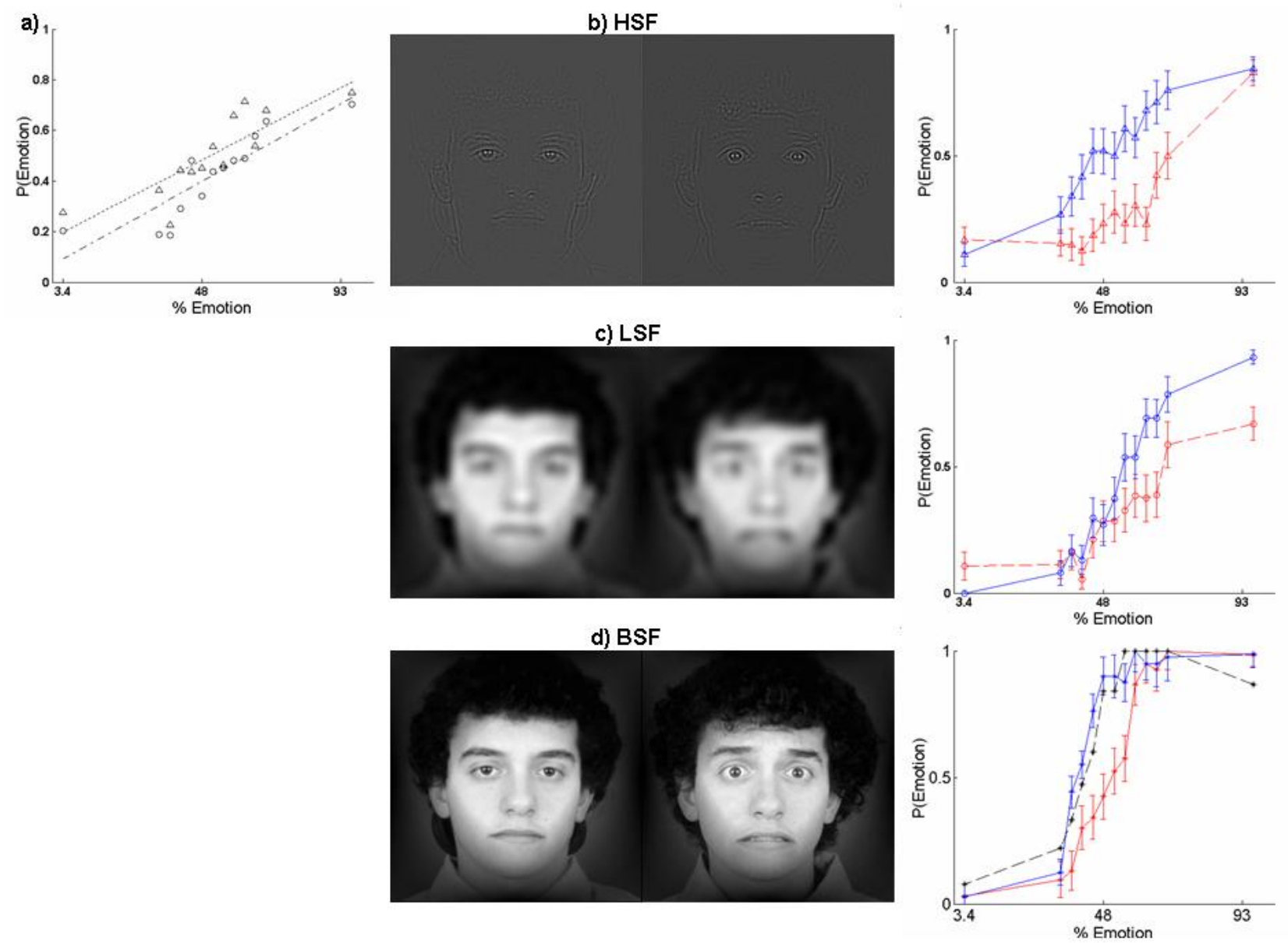

Fig. (5). Aftereffects with spatially filtered targets. To enable comparison with intact images (BSF), and the decision functions to the shorter duration targets in Fig. (6), we show all the decision functions to each different kind of target. Graph (a) shows the baseline condition without adaptation, with the average proportion of emotion reported with HSF targets (triangles, and dashed linear fit) and LSF targets (circles, dot-dashed fit), plotted against percent emotion in the target. Graphs (b-d) show the proportion of emotional responses after neutral (solid line) and emotional (dashed line) adaptors, plotted against the percent emotion of high (b), low (c) and broad (d) spatial frequency stimuli. The pre-adaptation baseline data (dotted line) in the BSF condition is also plotted in (d). Error bars are +/- S.E.M. In the centre is an example spatially filtered face shown in the three conditions. The graphs show a larger adaptation aftereffect with HSF targets, than with LSF targets. 
correlated with that expressed in the corresponding LSF set $(r\{27\}=0.35, p<0.05$, for single tailed t-test). Emotional adaptation in the HSF set was not correlated with that in the LSF set.

\section{Results: 23.5ms Adaptor}

The results of adaptation with a $23.5 \mathrm{~ms}$ adaptor are summarized in the right-most panels of Fig. (6). We find that significant aftereffects in HSF and LSF stimuli are correlated after a $23.5 \mathrm{~ms}$ adaptor, but that after the $11.7 \mathrm{~ms}$, only a weak residual aftereffect remains in the HSF targets. Subjects were $73 \%+/-16 \%$ (STD) accurate on the individual adaptors, significantly above chance (50\%) ( $t$-test $\mathrm{p}<.0001)$. The upper panels of Fig. (6) show a difference in the two adaptation conditions for both HSF and LSF targets.

A 3-way ANOVA with factors adaptor type (neutral, emotional and none) and morph level, and with subjects as a random effect, found a significant effect of adaptor type in both LSF $(F\{2,26\}=8.42, p<0.02, M S E=0.14)$ and HSF targets $(F\{2,26\}=3.24, p=0.05, M S E=0.108)$. One-tailed paired $t$-tests found the average aftereffect was less than zero for both HSF, $\mathrm{p}<0.05$, and LSF faces, $p<0.02$. The effect was slightly, but not significantly larger in the LSF targets $p=0.6$. The average within subject difference between emotional and baseline was also significantly less than zero, in both HSF $p<0.04$, and LSF $p<0.002$. The differences are represented on the bar chart in Fig. (6).

To examine the persistence of the aftereffects, we separated the baseline trials into those trials following an emotional adaptor trial, and those following a neutral trial, and found there was no significant difference between them. Accuracy on the endpoint images of the baseline (with no adaptation) was $82 \%+/-29 \%$ for HSF targets and $84 \%+/-26 \%$, for LSF.

The size of the adaptation aftereffect (i.e., the magnitude of the difference in responses between emotional and neutral adaptation in each subject) was strongly correlated between the HSF and LSF stimuli, $r\{13\}=0.92, p<0.0001$. This relationship is represented in the scatter graph in Fig. (6).

The extent to which subjects were able to categorize the adaptors (percent correct on the discrimination task) was also weakly correlated with the average adaptation effect in the HSF stimuli, $r=0.47, p=0.09$ and significantly correlated with the effect in LSF stimuli, $r=0.53 \mathrm{p}<0.05$. This correlation was largely driven by two outlying subjects, that showed the largest aftereffect, and near perfect adaptor discrimination. The correlation between the HSF and LSF effects, however,

\section{HSF: $23.5 \mathrm{~ms}$}

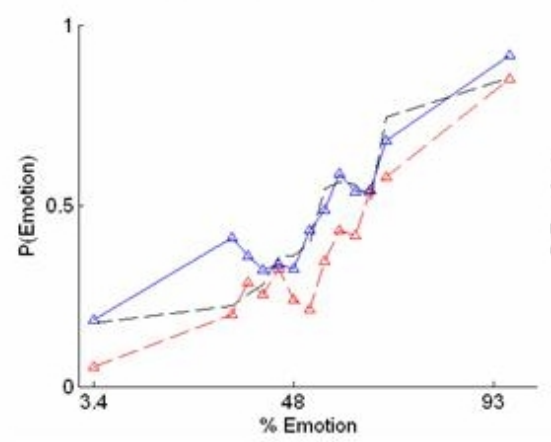

HSF: $11.7 \mathrm{~ms}$

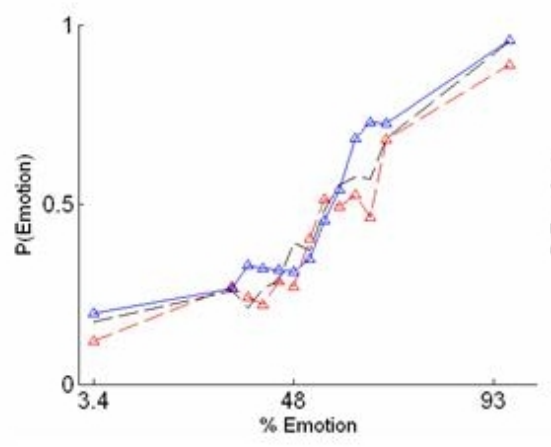

LSF: $23.5 \mathrm{~ms}$

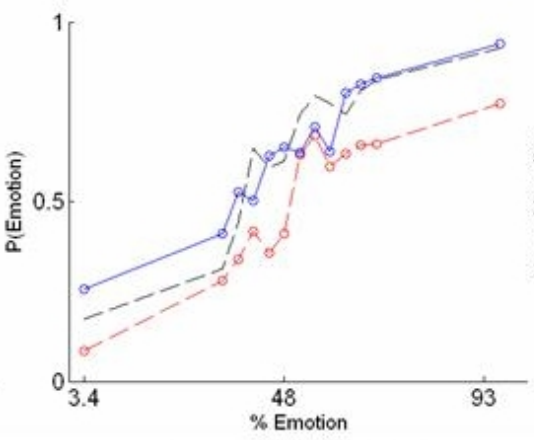

LSF: $11.7 \mathrm{~ms}$

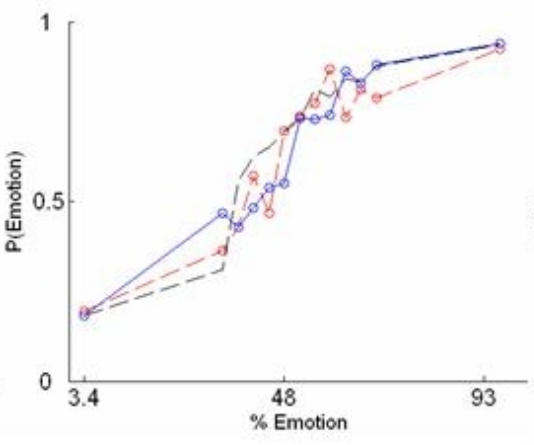

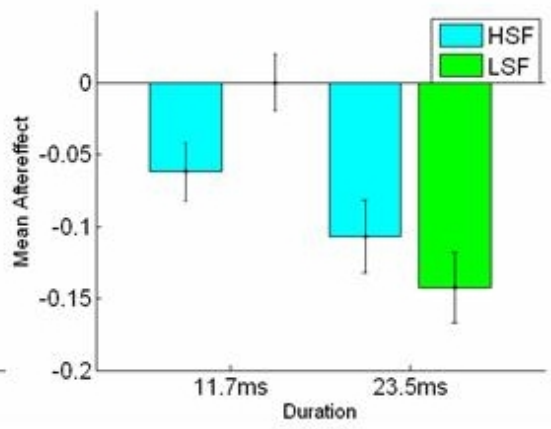

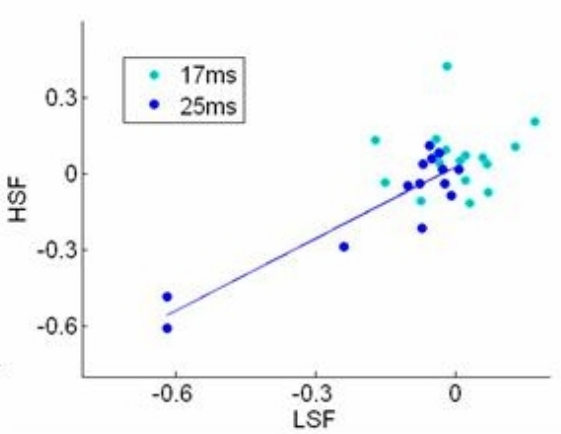

Fig. (6). Brief adaptors and spatially filtered targets.. The probability of emotional responses after a neutral adaptor (solid line) and an emotional adaptor (dashed line) plotted against percentage emotion in the target, shown for $23.5 \mathrm{~ms}$ adaptor durations (top), and for $11.7 \mathrm{~ms}$ durations (bottom) separated into HSF (triangles) and LSF (circles) targets. Errors are not shown, for the sake of clarity. The bar chart (top right) shows the mean aftereffects, as mean differences +/- S.E.M. A very weak mean residual aftereffect is still present in the HSF targets, with adaptor duration $11.7 \mathrm{~ms}$, smaller than the aftereffects for $23.5 \mathrm{~ms}$. The correlation between average adaptation of HSF and LSF targets at both durations (dark $23,5 \mathrm{~ms}$, light $11.7 \mathrm{~ms}$ ) for each subject is shown with the regression line for the fourteen subjects that saw adaptors of $23.5 \mathrm{~s}$ duration. 
survived the removal of those two points $(\mathrm{r}\{12\}=66$, $\mathrm{p}<0.03)$.

\section{Results: 11.7ms Adaptor}

The results for the $11.7 \mathrm{~ms}$ adaptor condition are shown on the bottom panels of Fig. (6). In the HSF targets, the aftereffect is now negligible, and no longer distributed evenly across the central morph levels. In the LSF targets, it is now non-existent.

Subjects were $71 \%+/-17 \%$ accurate on the adaptor discrimination trials, and therefore still statistically above chance $(p<0.001)$.

A 3-way ANOVA, fixed factors morph and adaptor category (neutral, emotional, and none), with subject as a random effect, found a no overall effect for HSF targets, $F\{2,32\}=2.59, p=0.09, M S E=0.13$, or LSF, $F\{2,32\}=1.95$, $p=0.16, M S E=0.016$. However, there was a residual effect for HSF faces, in the difference between neutral and emotional adapted responses, which was significantly less than zero in a one tailed t-test, $\mathrm{p}<0.03$. We also checked to see whether some individual subjects might show aftereffects, looking for a correlation between LSF and HSF adaptation effects, but this was now close to zero, $r=-0.08$.

\section{DISCUSSION}

The main purpose of these experiments was to test the possibility that rapid emotional processing through a LSF sensitive channel was driving the aftereffect at very short adaptor durations. The shortest duration that we expected to see a reliable aftereffect was $23.5 \mathrm{~ms}$, based on the results of experiment 2 but we included a shorter $11.7 \mathrm{~ms}$ duration as a control, to check for a residual, smaller effect in LSF targets.

We found no evidence of a channel specific effect in LSF at short durations, as the reliable aftereffects in LSF and HSF targets at $23.5 \mathrm{~ms}$ were strongly correlated within subjects. If anything, we found stronger effects in HSF, at both $11.7 \mathrm{~ms}$, and long (1s) durations. There are several explanations for this finding. One possibility is that adaptation of non face specific features, e.g. lines and curves, contributes to adaptation of HSF targets. Channel specific attention may also play a role in determining the level of adaptation to adaptors when there is a long period of exposure.

Although subjects were able to discriminate the content of both the $23 \mathrm{~ms}$, and the $11.7 \mathrm{~ms}$ adaptors, the effects on the following filtered targets were markedly different for the two durations. This is summarized in the last panel of Fig. (6).

A 'coarse to fine' hypothesis e.g., [56] suggests that LSF, or 'global' information is processed first, with HSF information, about the fine detail of image features, gradually added. Our results do not immediately support such a hypothesis. The shortest duration induces aftereffects on the categorization of HSF targets, rather than in LSF targets. A second possibility [55, 57-59] is that HSF information can also be accessed rapidly, but whether this occurs depends on the demands of the task. This explanation fits our data better, as one possibility is that the $11.7 \mathrm{~ms}$ presentation time, while below the threshold required to fully integrate information about the adaptor derived through different channels (if any), perhaps allows subjects to perform the discrimination task on the basis of some individual feature. A single feature - perhaps a high contrast feature such as the mouth, or the whites of the eyes, might be able to induce a rapid, very weak, "featural" aftereffect, that influences category decision [60], without necessarily inducing the category based (or, better, "whole image-based") aftereffect.

Further experiments are needed to investigate whether a single feature alone, such as eye shape or mouth shape, can induce an aftereffect at the category level, in the BSF image. We did not find evidence of an aftereffect at $11.7 \mathrm{~ms}$ in experiment 2 , but experiment 3 was a very much more sensitive design than the preceding experiments with multiple durations and conditions.

\section{GENERAL DISCUSSION}

The main findings of this paper are as follows. Experiment 1 found that the adaptation aftereffect magnitude depends on the duration of the adaptor, rather than on preceding exposure, and that significant adaptation could be induced with a $23.5 \mathrm{~ms}$ adaptor, very much shorter than anything previously reported. This is consistent with the short adaptation times used in complex shape adaptation, for example, by Suzuki and Cavanagh (1998).

In experiment 2 we also found that the aftereffect is sensitive to backward masking. We hypothesize that this sensitivity is best understood as an effect of subjects' accuracy at extracting category information from the adaptor. More speculatively, the findings are compatible with the possibility that part of the aftereffect is mediated by adaptation of relatively late neural firing, in high-level areas [61], consistent with translation invariance of the aftereffect to relatively short adaptors.

Our finding that adaptation to emotional content occurs at shorter durations than adaptation to identity content supports the idea that information about emotional content is available earlier and hence to some extent independently, of identity information. However, experiment 1 showed that at least part of the emotion aftereffect is identity specific. Moreover, the results of experiment 3 in the spatial frequency domain do not suggest that adaptation is mediated through independent spatial frequency specific pathways, as we did not observe a bias towards the adaptation of low spatial frequency information. We did notice a bias towards adaptation of HSF in experiment 3 , where we used relatively long, 1 second, adaptor durations.

One possible interpretation of the bias towards HSF is that high spatial frequency information becomes more important in relatively late occurring processing of faces. In particular, perhaps the integration of multiple features in HSF information requires more processing time. We note that one of the differences between the inverted face mask, and the quantized face mask, is the presence of more HSF information in the inverted face. It is possible that it is this late processing, or integration of HSF information that is interrupted by the strong masking in experiment 2 .

It is interesting that it appears to be masking the adaptor with a different face that interferes with the aftereffect, perhaps by competing for neural representational resources. Further work will establish whether non face salient, meaningful images can also produce this effect.

The results of experiment 2 also raised questions about the relationship between the explicit extraction of category 
information measured in the classification task, and the aftereffect. Our data are too sparse to allow us to say whether the two tasks depend differently on duration. The relationship between accuracy on the classification task, and aftereffect magnitude, does suggest that the two tasks use some of the same information. In further work we will consider the relationship at additional intermediate durations.

The final issue, which we deliberately do not raise in this paper, is the issue of the contribution of subjective awareness of the adaptor to aftereffect magnitude. We presume that awareness is correlated on average, with duration. Further investigation will uncover whether awareness of the adaptor on individual trials plays a role in aftereffect induction.

\section{ACKNOWLEDGEMENTS}

This work was supported by a HSFP grant RGP0047/2004-c to A.T. and we thank Ray Dolan for ideas about spatial frequency and Nick Furl for comments on an early version of the manuscript.

\section{Supplementary Material}

For experiment 1 we also include some additional discussion of methods in supplementary sections. It can be viewed at www.bentham.org/open/tobsj

\section{REFERENCES}

[1] Leopold DA, O'Toole AJ, Vetter T, et al. Prototype-referenced shape encoding revealed by high-level aftereffects. Nat Neurosci 2001; 4: 89-94.

[2] Webster MA, Kapling D, Mizokami Y, et al. Adaptation to natural facial categories. Nature 2004; 428: 557-61.

[3] Leopold DA, Rhodes G, Muller KM, et al. The dynamics of visual adaptation to faces. Proc Biol Sci 2005; 272: 897-904.

[4] Morrison DJ, Schyns PG. Usage of spatial scales for the categorization of faces, objects, and scenes. Psychon Bull Rev 2001; 8: 45469

[5] Harris CS, Gibson AR. Is orientation-specific color adaptation in human vision due to edge detectors, afterimages, or "dipoles"? Science 1968; 162: 1506-7.

[6] Paradiso MA, Shimojo S, Nakayama K. Subjective contours, tilt aftereffects, and visual cortical organization. Vision Res 1989; 29: 1205-13.

[7] Gibson JJ, Radner M. Adaptation, aftereffect and contrast in the perception of tilted lines I. Quantitative studies. J Exp Psychol 1937; 20: 453-67.

[8] Vul E, MacLeod DI. Contingent aftereffects distinguish conscious and preconscious color processing. Nat Neurosci 2006; 9: 873-4.

[9] Menghini F, van Rijsbergen N, Treves A. Modelling adaptation aftereffects in associative memory. Neurocomputing 2007; 70: 2000-4.

[10] Clifford CW, Webster MA, Stanley GB, et al. Visual adaptation: Neural, psychological and computational aspects. Vision Res 2007; 47: 3125-31.

[11] Webster MA, MacLin O. Figural aftereffects in the perception of faces. Psychon Bull Rev 1999; 6: 647-53.

[12] Yamashita JA, Hardy JL, De Valois K, et al. Stimulus selectivity of figural aftereffects for faces. J Exp Psychol Hum Percept Perform 2005; 31: 420-37.

[13] Hsu SM, Young A. Adaptation effects in facial expression recognition. Vis Cogn 2004; 11: 871-99.

[14] Watson TL, Clifford CW. Pulling faces: an investigation of the face-distortion aftereffect. Perception 2003; 32: 1109-16.

[15] Zhao L, Chubb C. The size-tuning of the face-distortion aftereffect. Vision Res 2001; 41: 2979-94.

[16] Rhodes G, Jeffrery L, Watson T, et al. Orientation-contingent face aftereffects and implications for face-coding mechanisms. Curr Biol 2004; 14: 2119-23.

[17] Kovacs G, Zimmer M, Harza I, et al. Position-specificity of facial adaptation. Neuroreport 2005; 16: 1945-9.
[18] Jiang F, Blanz V, O'Toole AJ. Probing the visual representation of faces with adaptation: A view from the other side of the mean. Psychol Sci 2006; 17: 493-500.

[19] Jiang F, Blanz V, O'Toole AJ. The role of familiarity in threedimensional view-transferability of face identity adaptation. Vision Res 2007; 47: 525-31.

[20] Rhodes G, Jeffrey L, Clifford DA, et al. The timecourse of higherlevel face aftereffects. Vision Res 2007; 47: 2291-6.

[21] Suzuki S, Cavanagh P. A shape-contrast effect for briefly presented stimuli. J Exp Psychol Hum Percept Perform 1998; 24: 1315-41.

[22] Fang F, Murray FO, Kersten D, et al. Orientation-tuned FMRI adaptation in human visual cortex. J Neurophysiol 2005; 94: 418895.

[23] Suzuki S. Attention-dependent brief adaptation to contour orientation: a high-level aftereffect for convexity? Vision Res 2001; 41: 3883-902.

[24] Kovacs G, Zimmer M, Harza I, et al. Adaptation duration affects the spatial selectivity of facial aftereffects. Vision Res 2007; 47 : 3141-3149.

[25] Bacon-Mace N, Mace MJM, Fabre-Thorpe M, et al. The time course of visual processing: backward masking and natural scene categorisation. Vision Res 2005; 4: 1459-69.

[26] Rolls ET, Tovee MJ, Purcell EG, et al. The responses of neurons in the temporal cortex of primates, and face identification and detection. Exp Brain Res 1994; 101: 473-84.

Kovacs G, Vogels R, Orban GA. Cortical correlate of pattern backward masking. Proc Natl Acad Sci USA 1995; 92: 5587-91.

[28] Humphreys GW, Bruce V. Visual cognition: computational, experimental, and neuropsychological perspectives. Hove Lawrence Erlbaum Associates 1989.

[29] Green MF, Glahn D, Engel SA, et al. Regional brain activity associated with visual backward masking. J Cogn Neurosci 2005; 17: 13-23.

[30] Fahrenfort JJ, Scholte HS, Lamme VA. Masking disrupts reentrant processing in human visual cortex. J Cogn Neurosci 2007; 19: 1488-97.

[31] Del Cul A, Baillet S, Dehaene S. Brain dynamics underlying the nonlinear threshold for access to consciousness. PLoS Biol 2007; 5: e260.

[32] Rolls ET, Tovee MJ, Panzeri S. The neurophysiology of backward visual masking: information analysis. J Cogn Neurosci 1999; 11: $300-11$

[33] Loffler G, Gordon GE, Wilkinson F, et al. Configural masking of faces: evidence for high-level interactions in face perception. Vision Res 2005; 45: 2287-97.

[34] Whalen PJ, Rauch SL, Etcoff EL, et al. Masked presentations of emotional facial expressions modulate amygdala activity without explicit knowledge. J Neurosci 1998; 18: 411-8.

[35] de Gelder B, Vroomen J, Portois G, et al. Non-conscious recognition of affect in the absence of striate cortex. Neuroreport 1999; 10 3759-63.

[36] Morris JS, Ohman A, Dolan RJ. Conscious and unconscious emotional learning in the human amygdala. Nature 1998; 393: 467-70.

[37] Morris JS, Friston KJ, Buchel C, et al. A neuromodulatory role for the human amygdala in processing emotional facial expressions. Brain 1998; 121: 47-57.

[38] Pizzagalli D, Regard M, Lehmann D. Rapid emotional face processing in the human right and left brain hemispheres: an ERP study. Neuroreport 1999; 10: 2691-8.

[39] Batty M, Taylor MJ. Early processing of the six basic facial emotional expressions. Brain Res Cogn Brain Res 2003; 17: 613-20.

[40] Pourtois G, Dan ES, Granjean D, et al. Enhanced extrastriate visual response to bandpass spatial frequency filtered fearful faces: time course and topographic evoked-potentials mapping. Hum Brain Mapp 2005; 26: 65-79.

[41] Eimer M, Holmes A. Event-related brain potential correlates of emotional face processing. Neuropsychologia 2007; 45: 15-31.

[42] Vuilleumier P, Armony JL, Clarke K, et al. Neural response to emotional faces with and without awareness: event-related fMRI in a parietal patient with visual extinction and spatial neglect. Neuropsychologia 2002; 40: 2156-66.

[43] Morris JS, deBonis M, Dolan RJ. Human amygdala responses to fearful eyes. Neuroimage 2002; 17: 214-22.

[44] Bar M. A cortical mechanism for triggering top-down facilitation in visual object recognition. J Cogn Neurosci 2003; 15: 600-9. 
[45] Dolan RJ, Vuilleumier P. Amygdala automaticity in emotional processing. Ann N Y Acad Sci 2003; 985: 348-55.

[46] Morris JS, Ohman A, Dolan RJ. A subcortical pathway to the right amygdala mediating "unseen" fear. Proc Natl Acad Sci USA 1999; 96: 1680-5.

[47] Matsumoto D, Ekman P. Japanese and Caucasian facial expressions of emotion (JACFEE) and neutral faces(JACNEUF). 1988

[48] Bachmann T, Luiga I, Poder E. Variations in backward masking with different masking stimuli: I. Local interaction versus attentional switch. Perception 2005; 34: 131-7.

[49] Costen NP, Shepard J, Ellis HD, et al. Masking of faces by facial and non facial stimuli. Vis Cogn 1994; 1: 227-251.

[50] Keysers C, Perrett D. Visual masking and RSVP reveal neural competition. Trend Cogn Sci 2002; 6: 120-25.

[51] Johnson M. Subcortical Face Processing. Nat Neurosci Rev 2005; 6: $766-74$.

[52] Winston JS, Vuilleumier P, Dolan RJ. Effects of low-spatial frequency components of fearful faces on fusiform cortex activity. Curr Biol 2003; 13: 1824-9.

[53] Vuilleumier P, Armony JL, Driver J, Dolan RJ. Distinct spatial frequency sensitivities for processing faces and emotional expressions. Nat Neurosci 2003; 6: 624-31.
[54] Gauthier I, Kurby K, Skudlasrski P, et al. Individual differences in FFA activity suggest independent processing at different spatial scales. Cogn Affect Behav Neurosci 2005; 5: 222-34.

[55] Schyns PG, Oliva A. Dr. Angry and Mr. Smile: when categorization flexibly modifies the perception of faces in rapid visual presentations. Cognition 1999; 69: 243-65.

[56] Goffaux V, Rossion B. Faces are "spatial"--holistic face perception is supported by low spatial frequencies. J Exp Psychol Hum Percept Perform 2006; 32: 1023-39.

[57] Ozgen E, Payne H, Sowden P, et al. Retinotopic sensitisation to spatial scale: evidence for flexible spatial frequency processing in scene perception. Vision Res 2006; 46: 1108-19.

[58] Oliva A, Schyns PG. Coarse blobs or fine edges? Evidence that information diagnosticity changes the perception of complex visual stimuli. Cognit Psychol 1997; 34: 72-107.

[59] Joyce CA, Schyns PG, Gosselin F, et al. Early selection of diagnostic facial information in the human visual cortex. Vision Res 2006; 46: $800-13$

[60] Williams MA, Moss SA, Bradshaw JL. A unique look at face processing: the impact of masked faces on the processing of facial features. Cognition 2004; 91: 155-72.

[61] Furl N, van Rijsbergen N, Treves A, et al. Experience-dependent coding of facial expression in superior temporal sulcus. Proc Natl Acad Sci USA 2007; 104: 13485-9.

(C) van Rijsbergen et al.; Licensee Bentham Open.

This is an open access article licensed under the terms of the Creative Commons Attribution Non-Commercial License (http://creativecommons.org/licenses/bync/3.0/) which permits unrestricted, non-commercial use, distribution and reproduction in any medium, provided the work is properly cited. 